\title{
1 A retrospective analysis of gender parity in scientific authorship in a 2 biomedical research centre
}

3 Rinita Dam $^{1^{*}+}$, rinita.dam@rdm.ox.ac.uk * Corresponding author

4 Syed Ghulam Sarwar Shah ${ }^{1}{ }^{2^{*}+}$, Sarwar.Shah@ouh.nhs.uk * Corresponding author

5 Maria Julia Milano ${ }^{1+}$, colomilano@gmail.com

6 Laurel D Edmunds ${ }^{1}$, laureledmunds@gmail.com

7 Lorna R Henderson ${ }^{1}{ }^{2}$, lorna.henderson@ouh.nhs.uk

8 Catherine R Hartley ${ }^{3}$, Catherine.hartley@bodleian.ox.ac.uk

9 Owen Coxall ${ }^{3}$, owen.coxall@bodleian.ox.ac.uk

10 Pavel V Ovseiko ${ }^{1}$, pavel.ovseiko@rdm.ox.ac.uk

11 Alastair M Buchan ${ }^{1}$, alastair.buchan@medsci.ox.ac.uk

12 Vasiliki Kiparoglou ${ }^{2,4}$, vasiliki.kiparoglou@ouh.nhs.uk

\section{Author institutional addresses}

$14{ }^{1}$ Radcliffe Department of Medicine. Medical Sciences Division, University of Oxford, Oxford, United 15 Kingdom.

$16{ }^{2}$ National Institute for Health Research Oxford Biomedical Research Centre, Oxford University 17 Hospitals NHS Foundation Trust, Oxford, United Kingdom.

$18{ }^{3}$ Bodleian Health Care Libraries, University of Oxford, Oxford, United Kingdom.

$19{ }^{4}$ Nuffield Department of Primary Health Care Sciences University of Oxford, Oxford.

$20+\mathrm{RD}$, SGSS and MJM contributed equally to this paper and are joint first authors on this work.

21 Word count: 4377 (excluding references) 


\section{ABSTRACT}

Objective: Scientific authorship is a vital marker of success in academic careers and gender equity is a key performance metric in research. However, there is little understanding of gender equity in publications in biomedical research centres funded by the National Institute for Health Research (NIHR). This study assesses the gender parity in scientific authorship of biomedical research.

Design: A retrospective descriptive study.

Setting: NIHR Oxford Biomedical Research Centre.

Data: 2409 publications accepted or published from 1 April 2012 to 31 March 2017.

Main outcome measures: Gender of authors, defined as a binary variable comprising either male or author, joint corresponding authors, last author and joint last authors.

Results: Publications comprised clinical research (39\%, $n=939$ ), basic research (27\%, $n=643$ ), and other types of research $(34 \%, n=827)$. The proportion of female authors as first author $(41 \%)$, first corresponding authors $(34 \%)$ and last author $(23 \%)$ was statistically significantly lower than male authors in these authorship categories. Of total joint first authors $(n=458)$, joint corresponding authors ( $n=169)$, and joint last authors ( $n=229)$, female only authors comprised statistically significant smaller proportions i.e. $15 \%(n=69), 29 \%(n=49)$ and $10 \%(n=23)$ respectively, compared to male only authors in these joint authorship categories. There was a statistically significant association between gender of the last author(s) with gender of the first author(s) $\left(\chi^{2} 33.742, \mathrm{P}<\right.$ $0.001)$, corresponding author(s) $\left(\chi^{2} 540.774, P<0.001\right)$ and joint last author $(s)\left(\chi^{2} 91.291, P<0.001\right)$.

43 Conclusions: Although there are increasing trends of female authors as first authors (41\%) and last 44 authors (23\%), female authors are underrepresented compared to male authors in all six categories 45 of scientific authorship in biomedical research. Further research is needed to encourage gender 46 parity in different categories of scientific authorship. 


\section{Strengths and limitations of this study}

48 - This is the first study to investigate gender parity in six categories of scientific authorship: first authors, first corresponding authors, last authors and three joint authorship categories i.e. joint first authors, joint corresponding authors and joint last authors in biomedical research.

- This study provides an important benchmark on gender equity in scientific authorship for other NIHR funded centres and organisations in England.

- The generalisability of the findings of this study may be limited due to differences in medical specialities, research areas, institutional cultures, and levels of support to individual researchers.

- Using secondary sources for determining the gender of authors may have limitations, which could be avoided by seeking relevant information from original authors and institution affiliation at the time of submission.

60

61 Keywords: Responsible Research and Innovation, Gender Equity, Scientific Authorship, National

62 Institution for Health Research, Biomedical Research Centres, Evaluation, Translational Research

63 Organisations, Translational Research, Basic Science Research. 


\section{INTRODUCTION}

65 Promoting Responsible Research and Innovation (RRI) is a major strategy of the "Science with and

66 for Society" work programme of the European Union's (EU) Horizon 2020 Framework Programme

67 for Research and Innovation[1]. RRI aims to build capacity and develop innovative ways to connect

68 science to society[2]. The RRI approach enables all societal members (such as researchers, citizens,

69 policymakers, businesses and third sector organisations) to work together during the research and innovation process in order to better align research and innovation with the values, needs and expectations of the society[1,2]. The RRI strategy includes the "keys" of public engagement, open access, gender, ethics and science education, and two further "keys": sustainability and social justice, which have been added recently [3]. The idea is that by prioritising these key components of $\mathrm{RRI}$, it would help make science more attractive to young people and society, and raise awareness of the meaning of responsible science[2].

We have focussed on the 'gender' element of the RRI because it is imperative to advance gender equality within research institutions, as well as within the design and content of research and innovation [1]. The issue of enhancing female participation in economic decision-making has become prominent in the national, European and international spheres, with a particular focus on the economic dimension of gender diversity[4]. In order to achieve a fair female participation within positions of power, it is recommended that women should hold half of the total seats in board rooms[5], however, a ratio between $40 \%$ and $60 \%$, also known as a "gender balance zone"[6], is considered acceptable $-\mathrm{a}$ threshold that is set by the European Commission[4].

From the perspective of gender equality in academia and scientific research, gender parity in scientific authorship is an important measure of achievement. The term gender parity refers to "the equal contribution of women and men to different dimensions of life" and it is operationalised as a "relative equality in terms of numbers and proportions of women and men" for a particular indicator"[7]. Gender (dis)parity in scientific authorship has important implications for gender equity in academic advancement[8] because scientific authorship is commonly used as a measure of academic productivity that is used for performance management, reward, and recognition $[9,10]$. The acceleration of women's advancement and leadership in research is one of the stated objectives of the National Institute of Health Research (NIHR) in the United Kingdom (UK) and it is imperative for the RRI in the wider European research area. Yet, there is limited research concerning gender equity in scientific authorship of translational research funded through NIHR biomedical research centres (BRCs).

In the UK, women currently outnumber men in medical schools[11], however, a persistent gender disparity in scientific publications remains[10,12-23]. While the proportion of women as first and senior authors of original medical research has increased over the past few decades[24], women are still significantly underrepresented as authors of research articles in medical journals, especially as first and senior authors[14,22,23,25]. For example, in radiology the proportion of women as first author increased from $8 \%$ in 1978 to $32 \%$ in 2013 and senior author increased from $7 \%$ in 1978 to $22 \%$ in 2013 [23]. Similarly, in gastroenterology the proportion of women as first author increased from $9 \%$ in 1992 to $29 \%$ in 2012, and senior author increased from 5\% in 1992 to $15 \%$ in 2012[14]. 
104 The profile of gender equity in higher education and research has been raised by the introduction of 105 Athena SWAN-linked funding incentives by the NIHR[26-28]. While Athena SWAN awards are useful 106 markers of achievement for higher education institutions and research institutes, they alone are 107 insufficient to assess and monitor the progress of NIHR BRCS towards gender equity[29]. Currently, 108 the proportion of women and the rate of their achievements are not tracked routinely by the NIHR 109 BRCs and little is known about how much women contribute to scientific research and innovation in 110 the BRCs. It is important to inform the acceleration of women's advancement and leadership in 111 translational research in line with the stated objectives of the NIHR within the UK and RRI within the 112 wider European research area through the collection of gender-disaggregated bibliometric data and 113 analysis of scientific authorship by gender.

114 For addressing the paucity of empirical research on women's advancement and leadership in 115 translational research in the UK and Europe, a recent study on gender equity in Neurology suggests 116 the need for institutions to take a systematic approach to addressing gender disparities that involve 117 customised, defined metrics and transparent reporting to stakeholders[30].

118 The aim of this study was to assess the gender parity in six types of scientific authorship in 119 biomedical research.

\section{METHODS}

\section{Study design}

122 A retrospective descriptive study.

\section{$123 \quad$ Setting}

124 This study was conducted at the NIHR Oxford BRC, which is research collaboration between the Oxford University Hospitals NHS Foundation Trust and the University of Oxford[31]. The aim of NIHR BRCs is to support translational research and innovation to improve healthcare for patients[32]. During the study period (April 2012-March 2017), the NIHR Oxford BRC was awarded $f 96 \mathrm{~m}$ to support research across nine research themes, five cross-cutting themes, and a range of underpinning platforms. The research themes included Blood, Cancer, Cardiovascular, Dementia and Cerebrovascular Disease, Diabetes, Functional Neuroscience and Imaging, Infection, Translational Physiology, and Vaccines. The crosscutting themes included Genomic Medicine, Immunity and Inflammation, Surgical Innovation and Evaluation, Biomedical Informatics and Technology, and Prevention and Population Care. The major underpinning platforms included a Biorepository, Education and Training, Public Engagement, and Research Governance. It is a contractual requirement to report the number of BRC supported publications published by researchers funded or supported by the NIHR research funds on an annual basis. Additionally, the NIHR uses bibliometric analyses to inform eligibility for NIHR funding[33,34]. This study was carried out as part of a wider programme of research on the markers of achievement for assessing and monitoring gender equity in translational research organisations[29]. 
140

\section{Data}

Data comprised translational research publications published by researchers funded or supported by the NIHR Oxford BRC. The eligibility criteria for inclusion of a publication were funding or support by the NIHR Oxford BRC and publication between April 2012 and March 2017. Based on these criteria, 2409 publications were identified. These publications were classified as: basic science studies, clinical studies (both trial and non-trial studies), and other studies (comments, editorials, systematic reviews, reviews, opinions, meeting reports, guidelines and protocols).

\section{Main outcome measures}

The main outcome measures were: (1) Gender of authors, defined as a binary variable comprising, either male or female categories, (2) Six categories of scientific authorship: first author, joint first authors, first corresponding author, joint corresponding authors, last author and joint last authors (Figure 1). These categories are conventionally associated with the highest amount of contribution, credit and prestige[10,17].

First author was defined as the first-named author of the publication. Publications that consisted of single authors were categorised as first authors. We considered the first author to be the main intellectual contributor in the publication, in terms of study design, data collection and analysis, and manuscript writing. Joint first authors were defined as two or more authors who were named as equal contributors and mentioned as joint first authors of the publication. The first corresponding author was defined as the only author who was reported as a corresponding author in the publication and his/her contact details such as an institutional address and/or an email address were provided for correspondence in the publication. Joint corresponding authors were defined as two or more authors who were listed or marked as corresponding authors and their contact details were provided for correspondence in the publication. Last author was defined as the last-named author of a publication. The last author was considered to be a group leader or principal investigator who may have provided significant intellectual contribution or supervision of the research work as well as acquisition of research funding[17,35]. Joint last authors were defined as two or more authors who were named as equal contributors in the publication and their names were mentioned as joint last authors in the publication. A major confounding factor, for which we could not control, was the informal nature of the conventions for the sequence and role of authors[35]. Although conventions for scientific authorship are well established in biomedical sciences[36,37], they may vary between different research areas and even between different research groups within the same area.

\section{Determination of gender of authors}

The gender of the authors was defined as a binary variable comprising either male or female categories, which were determined based on the first name of authors in all six categories of authorship included in the analysis. When the first names of authors were initialled in the publication or were difficult to associate with either male or female gender, further information was sought through searching their institutional webpages and online social network sites such as the LinkedIn and ResearchGate. We also used the Gender API (gender-api.com) when it was not possible to ascertain the gender of the authors by the above-mentioned sources. In addition, we contacted five authors directly via email to ascertain their gender. After completing data coding by two researchers (MJM and RD), to ensure the accuracy of data coding, $10 \%$ of the data were checked 
181

182

183

184

185

186

187

188

189

190

191

192

193

194

195

196

197

198

199

200

201

202

203

204

205 independently $(\mathrm{CH})$. Consensus was achieved through discussion between the researchers on data fields that did not match the assigning of the gender of authors and types of authorship (Figure 1).

\section{Statistical analysis}

Data were analysed using frequencies including counts and percentages. Chi-square tests were used for identifying statistically significant differences and associations between male and female authors in various categories of authorship. The level of significance was set at $p<0.05$. Data were analysed using the IBM SPSS Statistics for Windows, Version 25.0 (Armonk, NY: IBM Corp.).

\section{Patient and public involvement statement}

There was no patient or public involvement in the study design.

\section{RESULTS}

\section{Type of research study}

Table 1 presents an overview of the types of research studies by year. Clinical research studies (both trial and non-trial studies) comprised $39 \%$ ( $n=939$ ), basic science research $27 \%$ ( $n=643$ ) and a third of publications $(34 \%, n=827)$ included other types of research, such as systematic reviews, reviews, research protocols, editorials, guidelines, opinions, comments, and meeting reports.

Table 1 Number of publications by year of acceptance and types of research studies

\begin{tabular}{|c|r|r|r|r|r|}
\cline { 2 - 6 } \multicolumn{1}{c|}{} & \multicolumn{4}{c|}{ Types of research studies } \\
Count (\%) & \multicolumn{1}{c|}{} \\
\hline $\begin{array}{c}\text { Year } \\
\text { (Accepted) }\end{array}$ & $\begin{array}{c}\text { Basic } \\
\text { science }\end{array}$ & $\begin{array}{c}\text { Clinical } \\
\text { trial }\end{array}$ & $\begin{array}{c}\text { Clinical study - } \\
\text { Not a trial }\end{array}$ & \multicolumn{1}{c|}{ Other* } & \multirow{2}{*}{$\begin{array}{c}\text { Total Count } \\
\text { (\%) }\end{array}$} \\
\hline $2012 \dagger$ & $75(27.6)$ & $18(6.6)$ & $90(33.1)$ & $89(32.7)$ & $272(100)$ \\
\hline 20130 & $151(28.2)$ & $27(5.0)$ & $183(34.2)$ & $174(32.5)$ & $535(100)$ \\
\hline 20140 & $122(22.2)$ & $29(5.3)$ & $204(37.2)$ & $194(35.3)$ & $549(100)$ \\
\hline 20150 & $137(24.7)$ & $48(8.7)$ & $158(28.5)$ & $211(38.1)$ & $554(100)$ \\
\hline 20160 & $137(31.8)$ & $31(7.2)$ & $120(27.8)$ & $143(33.2)$ & $431(100)$ \\
\hline $2017 \ddagger$ & $21(30.9)$ & $5(7.4)$ & $26(38.2)$ & $16(23.5)$ & $68(100)$ \\
\hline Total & $643(26.7)$ & $158(6.6)$ & $\mathbf{7 8 1 ( 3 2 . 4 )}$ & $\mathbf{8 2 7}(34.3)$ & $\mathbf{2 4 0 9 ( 1 0 0 )}$ \\
\hline
\end{tabular}

†April-December, ৩January-December ‡January-March, *systematic reviews, reviews, research protocols, editorials, guidelines, opinions, comments, and meeting reports

\section{Authorship type and Gender}

Table 2 presents an overview of gender of authors by types of authorship. This highlights that male authors were statistically significant more likely to be first authors (59\%, $\chi 2$ 972.938, $P<0.001)$, first corresponding authors $\left(66 \%, \chi_{2} 242.970, \mathrm{P}<0.001\right)$ and last authors $(77 \%, \chi 2702.411, \mathrm{P}<0.001)$ ) (Table 2). Furthermore, analyses of joint authorship categories revealed that the proportion of 'female only' authors was statistically significantly lower than 'male only' authors in the joint corresponding authors $\left(29 \%, \chi_{2} 79.858, \mathrm{P}<0.001\right)$ and joint last authors categories $\left(10 \%, \chi_{2} 56.550\right.$, 
$206 \mathrm{P}<0.001$ ) (Table 2). However, in the joint first authors category, the proportion of 'male and female'

207 as joint first authors $(57 \%, \chi 2128.467, \mathrm{P}<0.001)$ was statistically significantly higher than male only

208 and female only first authors (Table 2 ).

209 Table 2. Authorship categories and gender of authors

\begin{tabular}{|l|l|l|l|l|}
\hline \multirow{2}{*}{$\begin{array}{l}\text { Nuthorship type } \\
\text { Number of publications in the category }\end{array}$} & \multicolumn{3}{|c|}{ Count (\%) } & \multicolumn{1}{|c|}{$\chi^{2}(p)$} \\
\hline & Male only & Female only & Male and female & \\
\hline First author ( $n=2407)$ & $1413(58.7)$ & $994(41.3)$ & N/A* & $72.938(<0.001)$ \\
\hline First corresponding author $(n=2371)$ & $1565(66)$ & $806(34)$ & N/A & $242.970(<0.001)$ \\
\hline Last author ( $n=2406)$ & $1853(77)$ & $553(23)$ & N/A & $702.411(<0.001)$ \\
\hline Joint first authors $(n=458)$ & $127(27.7)$ & $69(15.1)$ & $262(57.2)$ & $128.467(<0.001)$ \\
\hline Joint corresponding authors $(n=169)$ & $107(63.3)$ & $49(29)$ & $13(7.7)$ & $79.858(<0.001)$ \\
\hline Joint last authors $(n=229)$ & $108(47.2)$ & $23(10)$ & $98(42.8)$ & $56.550(<0.001)$ \\
\hline
\end{tabular}

${ }^{*} \mathrm{~N} / \mathrm{A}=$ not applicable.

210 Gender of authors by type of research studies

211 Analysis of gender of authors by types of research studies (i.e. basic science, clinical trials, non-trial

212 clinical studies and other research) showed that the proportions of male only authors were

213 statistically significantly higher than the proportions of female only authors in three authorship

214 categories: first authors ( $\chi 28.606$ ( $d f 3$ ), $P=0.035$ ), first corresponding authors ( $\chi 236.955$ (df 9 ), $P<$

2150.001 ) and last authors ( $\chi 210.314$ (df 3 ), $P=0.016$ ). The analysis by type of research studies also

216 revealed that there were no significant differences between the proportions of male only and

217 female only authors in all three joint authorship categories: joint first authors ( $\chi 25.549$ (df 6), $P=$

218 0.476), joint corresponding authors ( $\chi 29.021$ ( $d f 6), P=0.172$ ) and joint last authors ( $\chi 28.433$ (df 6),

$219 \quad P=0.208)$.

\section{Yearly trends in Authorship by gender}

221 Figure 2 presents the yearly trends in scientific authorship by gender. In all authorship types and

222 across all five years of publication, the proportion of male and female authors varied (Figure 2). The

223 analysis showed women were significantly underrepresented across all years and authorship types.

224 Interestingly, joint first authorship indicated a higher proportion of 'male and female' authors

225 compared to 'male only' and 'female only' authors (Figure 2). 


\section{Association between same gender across authorship categories}

227 There was a statistically significant association between the same gender in first authorship and 228 corresponding authorship categories $\left(\chi^{2} 775.425\right.$ (df 3$\left.), P<0.001\right)$ and the first author and joint first 229 authors $\left(\chi^{2} 138.849\right.$ (df 2$\left.), P<0.001\right)$.

230 Furthermore, there were statistically significant associations between the same gender in the last 231 author category with the same gender of first author(s) ( $\chi^{2} 33.742$ (df 2), $P<0.001$ ), corresponding 232 author(s) ( $\chi^{2} 540.774$ (df 1$), P<0.001$ ) and joint last authors ( $\chi^{2} 91.291$ (df 2$\left.), P<0.001\right)$. However, 233 there was no statistically significant association between the male and female last authors with the 234 respective gender of joint first authors $\left(\chi^{2} 4.29(\mathrm{df} 2), P=0.117\right)$.

\section{DISCUSSION}

236 We retrospectively analysed the gender parity of authors in six categories of authorship of scientific publications that were published over a five-year period. Our analysis shows that the number of female authors were underrepresented across all six categories of authorship $[10,38,39]$. In the first author category the proportions of female authors and male authors were within the $40 \%-60 \%$ "gender balance zone"[6]. The greatest gender imbalance was observed in the last author category where 'female only' authors comprised only $23 \%$. Nonetheless, this proportion is higher than other studies reporting similar analyses[11,16,24].

To the best of our knowledge, this study presents the first analysis of joint authorship in three categories. Secondly, it demonstrates underrepresentation in female only authors in six categories of scientific authorship[40]. Thirdly, the analysis highlights gender inequity with female underrepresentation in prestigious authorship positions compared to male in biomedical research. This is consistent with other fields including: epilepsy, lung cancer, dermatology, eating disorders and in medicine in general[17,19,41-43].

This study extends understanding of gender-based trends in scientific authorship (Figure2) by showing encouraging incremental changes in gender parity in authorship in a biomedical research setting. Previous research examined the gender gap in authorship within the medical literature reporting an upward trend for female first authors from $6 \%$ in 1970 to $29 \%$ in 2004 and female last authors from $4 \%$ in 1970 to $19 \%$ 2004. However, it was limited to US based institutions[12]. A similar first authors increasing from $11 \%$ in 1970 to $37 \%$ in 2004 and female last authors from $12 \%$ in 1970 to $17 \%$ in 2004 [24]. In addition, a recent study by Filardo et al.[16] examined the prevalence of female first authorship of original research published in six high impact general medical journals between February 1994 and June 2014 revealed that the adjusted probability of an article having a female first authorship increased significantly from $27 \%$ in 1994 to $37 \%$ in $2014[16]$. However, despite the proportion of female first authors varied greatly by journal, men were generally more likely to be first authors than women[16]. Compared to previous studies mentioned above, our study provides evidence of higher and increasing gender equity in the first authors, last authors and other four categories of scientific authorship in biomedical research (Table 2). 
264 Our study identified a strong association between same gender and authorship types showing if the

265 first author of a publication was male, it was highly likely that the first corresponding author of the same publication would also be male. Similarly, the likelihood of the first author being female was higher, if the first corresponding author was also female[44]. Likewise, there appeared to be a significant association of male and female last authors with the respective gender of first authors. Previous research has highlighted males and females were more likely to be first authors on papers if the last authors were of the same gender; however, these were not conducted in a translational research setting[23,45-47]. There was also a strong association of male and female last authors with the respective gender of corresponding authors[44].

However, due to the differences in gender equity between different research areas and medical specialties, where a centre-specific mix of research themes is likely to influence gender equity in scientific authorship, it is difficult to make direct comparisons across the literature.

Overall, our results build an important evidence base in biomedical research settings concerning gender parity and support the findings from previous studies where analysis of scientific authorship by gender has been used as an important marker of gender equity[12,24,48-50].

\section{Implications for policy and practice}

While NIHR BRCS routinely collect bibliometric data on publications arising from the NIHR-funded research, and report to the NIHR (the funder), to the best of our knowledge, this data is not routinely analysed by gender. Our study supports the feasibility of using NIHR BRCs funded or supported research publications for analysing scientific authorship by gender. While retrospective analysis of the gender of authors in scientific publications is labour-intensive and has limitations, there is an opportunity to begin to track this prospectively. As more data becomes available, this would enable longitudinal analysis of scientific authorship by gender, which could be useful for tracking progress towards gender equity and related issues such as markers of achievement across all NIHR BRCs.

In addition, since the acceleration of women's advancement and leadership in translational research is one of the stated objectives of the NIHR, investigating the extent of gender equity in scientific authorship may usefully inform strategies to accelerate women's advancement and leadership in NIHR-funded research. Moreover, bibliometric analyses used by the NIHR to inform competition for NIHR funding may incorporate the gender dimension into the analysis, which could provide additional information on the competitiveness for NIHR funding[51,52].

\section{CONCLUSION}

Our results show that while first authorship is within the $40 \%-60 \%$ gender balance zone, a greater gender disparity is prevalent in other types of scientific authorship in biomedical research. The proportion of female authors is significantly lower than the proportion of male authors in all six categories of authorship included in our analysis. This study also demonstrates the feasibility of analysing scientific authorship by gender, which could provide useful insight about gender equity in scientific publications, which may be a useful marker of achievement. Overall, our study demonstrates that it is feasible to analyse the available bibliometric data on publications arising 
from NIHR funding by gender and consider establishing processes for analysing gender equity in scientific authorship over time.

\section{Contributors}

LDE conceived the study. RD and MJM coded the data. SGSS analysed the data. RD and SGSS drafted the manuscript. PVO contributed to the conception of the study and co-wrote parts of the manuscript. $\mathrm{CRH}$ and $\mathrm{OC}$ participated in data collection. VK, $\mathrm{LRH}$, and $\mathrm{AMB}$ contributed to the conception of the study, facilitated access to the publications and coordinated the study. All authors read, contributed to and approved the final manuscript.

\section{Funding}

This study is funded by the European Union's Horizon 2020 research and innovation programme award STARBIOS2 under grant agreement No. 709517 and by the National Institute for Health Research (NIHR) Oxford Biomedical Research Centre, grant BRC-1215-20008 to the Oxford University Hospitals NHS Foundation Trust and the University of Oxford. The views expressed are those of the authors and not necessarily those of the NHS, the NIHR, or the Department of Health and Social Care.

\section{Competing interests}

VK is Chief Operating Officer and LRH is Clinical Research Manager at the National Institute for Health Research (NIHR) Biomedical Research Centre, Oxford. AMB is a senior medical science advisor and co-founder of Brainomix, a company that develops electronic ASPECTS (e-ASPECTS), an automated method to evaluate ASPECTS in stroke patients. MJM, LDE and PVO were funded by STARBIOS2 and the National Institute for Health Research (NIHR) Oxford Biomedical Research Centre (BRC). PVO is a member of the NIHR Advisory Group on Equality, Diversity, and Inclusion, a member of the European Association of Science Editors Gender Policy Committee, and a member of the Athena SWAN Self-Assessment Team of the Radcliffe Department of Medicine, University of Oxford. The other authors declare no competing interests.

\section{Ethics}

The University of Oxford Clinical Trials and Research Governance Team reviewed the study and deemed it exempt from full ethics review on the grounds that it falls outside of the Governance Arrangements for Research Ethics Committees (GAfREC), which stipulate which research studies are required to have ethics review. A wider programme of research on the activities of the NIHR Oxford Biomedical Research Centre from 2017 to 2022 received ethics clearance through the University of Oxford Central University Research Ethics Committee (R51801/ RE001), the Health Research Authority (IRAS ID 228049) and the Oxford University Hospitals NHS Foundation Trust Management (PID 12779).

\section{Acknowledgements}

The authors wish to thank Professor Helen McShane for reviewing the draft manuscript and making comments and suggestions for improving it. 


\section{Patient consent for publication}

341 Not required.

\section{Data sharing statement}

343 No additional data available.

\section{REFERENCES}

3451 Colizzi V, Mezzana D, Ovseiko P V., et al. Structural transformation to attain responsible 346 BIOSciences (STARBIOS2): Protocol for a horizon 2020 funded European multicenter project to promote responsible research and innovation. J Med Internet Res 2019;21. doi:10.2196/11745

Elster D, Barendziak T, Birkholz J. Towards a Sustainable and Open Science: Recommendations for Enhancing Responsible Research and Innovation in the Biosciences at the University of Bremen. 2019. http://elib.suub.uni-bremen.de/edocs/00107721-1.pdf

European Commission. Indicators for promoting and monitoring Responsible Research and Innovation. Report from the Expert Group on Policy Indicators for Responsible Research and Innovation. 2015.

European Commission. DIRECTIVE OF THE EUROPEAN PARLIAMENT AND OF THE COUNCIL on improving the gender balance among non-executive directors of companies listed on stock exchanges and related measures. Published Online First: 2012.http://eurlex.europa.eu/LexUriServ/LexUriServ.do?uri=COM:2012:0614:FIN:en:PDF

5 European Commission. Report on Equality between Women and Men in the European Union. 2019;:1-78. doi:10.2838/776419

Kuhlmann E, Ovseiko P V., Kurmeyer C, et al. Closing the gender leadership gap: A multicentre cross-country comparison of women in management and leadership in academic health centres in the European Union. Hum Resour Health 2017;15:1-7. doi:10.1186/s12960016-0175-y

7 European Institute for Gender Equality. Gender parity. 2020.https://eige.europa.eu/thesaurus/terms/1195 (accessed 19 Feb 2020).

8 Rexrode KM. The gender gap in first authorship of research papers. BMJ 2016;1130:1-2. doi:10.1136/bmj.i1130

9 Bloch C, Graversen EK, Pedersen HS. Competitive Research Grants and Their Impact on Career Performance. Minerva 2014;52:77-96.

10 Bendels, M, Muller, R, Brueggmann, D, Groneberg D. Gender disparities in high-quality research revealed by Nature Index journals. PLoS One 2018;13:1-21.

11 Jefferson L, Bloor K, Maynard A. Women in medicine: Historical perspectives and recent trends. Br Med Bull 2015;114:5-15. doi:10.1093/bmb/ldv007

12 Jagsi, R, Guancial, E, Worobey, C, Henault, L, Chang, Y, Starr, R, Tarbell, N, Hylek E. The "Gender Gap" in Authorship of Academic Medical Literature - A 35-Year Perspective. N Engl 
J Med 2006;355:281-7.

13 Mueller CM, Gaudilliere DK, Kin C, et al. Gender disparities in scholarly productivity of US academic surgeons. J Surg Res 2016;203:28-33. doi:10.1016/j.jss.2016.03.060

14 Long MT, Leszczynski A, Thompson KD, et al. Female authorship in major academic gastroenterology journals: A look over 20 years. Gastrointest Endosc 2015;81:1440-1447.e3. doi:10.1016/j.gie.2015.01.032

Fishman M, Williams WA, Goodman DM, et al. Gender Differences in the Authorship of Original Research in Pediatric Journals, 2001-2016. J Pediatr 2017;191:244-249.e1. doi:10.1016/j.jpeds.2017.08.044

16 Filardo G, Graca B, Sass DM, et al. Trends and comparison of female first authorship in high impact medical journals??: observational study ( 1994-2014 ). BMJ 2016;352:1-8. doi:10.1136/bmj.i847

Bendels, Michael, Wanke, Eileen, Schoffel, Norman, Bauer, J, Quarcoo, David and Groneberg D. Gender equality in academic research on epilepsy - a study on scientific authorships. Epilepsia 2017;58:1794-802. doi:10.1111/epi.13873

18 Bendels MHK, Brüggmann D, Schöffel N, et al. Gendermetrics of cancer research?: results from a global analysis on lung cancer. Oncotarget $2017 ;: 1-11$.

19 Bendels MHK, Dietz MC, Brüggmann D, et al. Gender disparities in high-quality dermatology research[: a descriptive bibliometric study on scientific authorships. BMJ Open 2018;8:1-11. doi:10.1136/bmjopen-2017-020089

20 Bendels MHKK, Brüggmann D, Schöffel N, et al. Gendermetrics of cancer research: Results from a global analysis on prostate cancer. Oncotarget 2018;9:19640-9. doi:10.18632/oncotarget. 24716

21 Sing DC, Jain D, Ouyang D. Gender trends in authorship of spine-related academic literature - a 39-year perspective. Spine J 2017;17:1749-54. doi:10.1016/j.spinee.2017.06.041

Schisterman EF, Swanson CW, Lu Y, et al. The changing face of epidemiology: gender disparities in citations? Epidemiology 2017;28:159-68. doi:10.1097/EDE.0000000000000593.The

23 Piper $\mathrm{CL}$, Scheel JR, Lee $\mathrm{Cl}$, et al. Gender trends in radiology authorship: A 35-year analysis. Am J Roentgenol 2016;206:3-7. doi:10.2214/AJR.15.15116

24 Sidhu R, Rajashekhar P, Lavin VL, et al. The gender imbalance in academic medicine: A study of female authorship in the United Kingdom. J R Soc Med 2009;102:337-42. doi:10.1258/jrsm.2009.080378

25 Sing DC, Jain D, Ouyang D. Gender trends in authorship of spine-related academic literaturea 39-year perspective. Spine J 2017;17:1749-54. doi:10.1016/j.spinee.2017.06.041

26 Ovseiko P V., Chapple A, Edmunds LD, et al. Advancing gender equality through the Athena SWAN Charter for Women in Science: An exploratory study of women's and men's perceptions. Heal Res Policy Syst 2017;15:1-13. doi:10.1186/s12961-017-0177-9

Ovseiko P V., Pololi LH, Edmunds LD, et al. Creating a more supportive and inclusive university 
culture: a mixed-methods interdisciplinary comparative analysis of medical and social sciences at the University of Oxford. Interdiscip Sci Rev 2019;44:166-91. doi:10.1080/03080188.2019.1603880

Schmidt EK, Ovseiko P V, Henderson LR, et al. Understanding the Athena SWAN award scheme for gender equality as a complex social intervention in a complex system: analysis of Silver award action plans in a comparative European perspective. bioRxiv 2019;:555482. doi:10.1101/555482

29 Ovseiko, PV, Edmunds, LD, Pololi, LH et al. Markers of achievement for assessing and monitoring gender equity in translational research organisations?: a rationale and study protocol. BMJ Open 2016;6:1-11. doi:10.1136/bmjopen-2015-009022

Silver JK. Understanding and addressing gender equity for women in neurology. Neurology 2019;93:538-49. doi:10.1212/WNL.0000000000008022

NIHR Oxford BRC. About the NIHR Oxford Biomedical Research Centre. 2019.https://oxfordbrc.nihr.ac.uk/about-us-intro/ (accessed 19 Dec 2019).

Greenhalgh T, Ovseiko P V., Fahy N, et al. Maximising value from a United Kingdom Biomedical Research Centre: study protocol. Heal Res policy Syst 2017;15:70. doi:10.1186/s12961-017-0237-1

34 Gunashekar S, Wooding S, Guthrie S. How do NIHR peer review panels use bibliometric information to support their decisions? Scientometrics 2017;112:1813-35. doi:10.1007/s11192-017-2417-8

Gunashekar S, Parks S, Calero-Medina C, et al. Bilbliometric analysis of highly cited publications of biomedical and health research in England, 2004--2013. Bilbliometric Anal highly cited Publ Biomed Heal Res England, 2004--2013 2016;:83. doi:https://doi.org/10.7249/RR1363

36 Wren JD, Kozak KZ, Johnson KR, et al. The write position. A survey of perceived contributions to papers based on byline position and number of authors. EMBO Rep 2007;8:988-91. doi:10.1038/sj.embor.7401095

ICMJE. Defining the Role of Authors and Contributors. 2019.http://www.icmje.org/recommendations/browse/roles-and-responsibilities/definingthe-role-of-authors-and-contributors.html (accessed 23 Dec 2019).

38 Holman L, Stuart-fox D, Hauser CE. The gender gap in science: How long until women are equally represented? PLOS Biol 2018;16:1-20. doi:10.1371/journal.pbio.2004956

UNESCO. Women in Science. Fact Sheet. 2018.

40 Broderick NA, Casadevall A. Gender inequalities among authors who contributed equally. Elife 2019;8:1-11. doi:10.7554/eLife.36399

41 Bendels MHK, Bruggmann D SN et al. Gendermetrics of cancer research: results from a global analysis on lung cancer. Oncotarget 2017;:1-11. 
42 Strand M, Bulik CM. Trends in female authorship in research papers on eating disorders: 20year bibliometric study. BJPsych Open 2018;4:39-46. doi:10.1192/bjo.2017.8

43 Gayet-Ageron A, Poncet A, Perneger T. Comparison of the contributions of female and male authors to medical research in 2000 and 2015: A cross-sectional study. BMJ Open 2019;9:1-8. doi:10.1136/bmjopen-2018-024436

44 Ouyang, D, Harrington, RA., Rodriguez F. Association Between Female Corresponding Authors and Female Co-Authors in Top Contemporary Cardiovascular Medicine Journals. Circ Am Hear Assoc 2019;139:1127-9. doi:10.1161/CIRCULATIONAHA.118.037763

45 Fox CW, Burns CS, Muncy AD, et al. Gender differences in patterns of authorship do not affect peer review outcomes at an ecology journal. Funct Ecol 2016;30:126-39. doi:10.1111/13652435.12587

46 Salerno PE, Páez-Vacas M, Guayasamin JM, et al. Male principal investigators (almost) don't publish with women in ecology and zoology. PLoS One 2019;14:1-14. doi:10.1371/journal.pone.0218598

47 Edwards HA, Schroeder J, Dugdale HL. Correction: Gender differences in authorships are not associated with publication bias in an evolutionary journal(PLoS ONE (2019)13:8 (e0201725)Doi:10.1371/journal.pone.0201725). PLoS One 2019;14:1-16. doi:10.1371/journal.pone.0217251

48 Bendels MHK, Müller R, Brueggmann D, et al. Gender disparities in high-quality research revealed by nature index journals. PLoS One 2018;13:1-21. doi:10.1371/journal.pone.0189136

49 Feramisco JD, Leitenberger JJ, Redfern SI, et al. A gender gap in the dermatology literature?? Cross-sectional analysis of manuscript authorship trends in dermatology journals during 3 decades. J Am Dermatology 2009;60:63-9. doi:10.1016/j.jaad.2008.06.044

50 Bhattacharyya N, Shapiro NL. Increased Female Authorship in Otolaryngology Over the Past Three Decades. Laryngoscope 2000;110:358-61. doi:10.1097/00005537-200003000-00005

51 Van Leeuwen T, Hoorens S, Grant J. Using bibliometrics to support the procurement of NIHR biomedical research centres in England. Res Eval 2009;18:71-82. doi:10.3152/095820209X414178

52 Leeuwen TVan, Grant J. Bibliometric analysis of highly cited publications of health research in England, 1995-2004. 2007. https://www.rand.org/pubs/working_papers/WR368.html

\section{Additional files list}

Figure 1 Publication analysis workflow. The workflow shows the process of extracting data according to gender from six types of authorship.

Figure 2 Yearly trends in scientific authorship by gender (male and female), April 2012 - March 2017. This plot represents the yearly variation of the proportion of male and female authors according to six types of authorship between the years of publication/acceptance from 2012 to 2017. 
bioRxiv preprint doi: https://doi.org/10.1101/2020.02.24.962175; this version posted February 25, 2020. The copyright holder for this preprint (which was not certified by peer review) is the author/funder, who has granted bioRxiv a license to display the preprint in perpetuity. It is made available under aCC-BY-NC-ND 4.0 International license.

\section{Supplementary File 1}

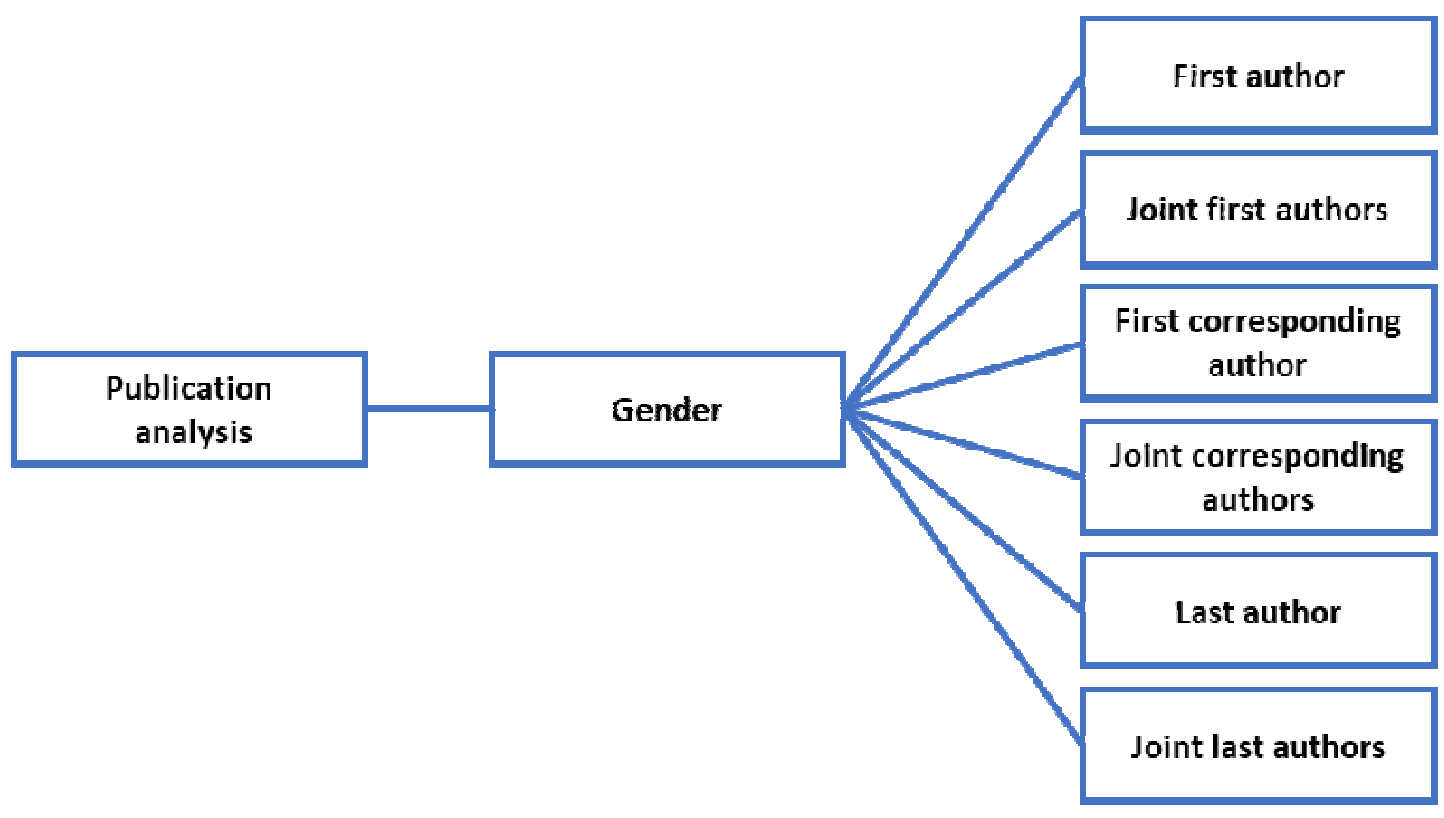

Figure 1 Publication analysis workflow. 